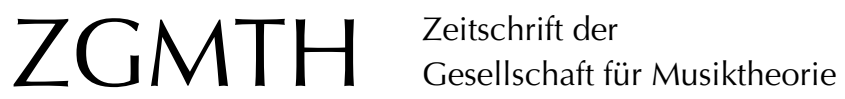

Neidhöfer, Christoph (2003/05): Twelve-Tone Theory. ZGMTH 1-2/2/2-3, 207-217. https://doi.org/10.31751/529

(C) 2003/05 Christoph Neidhöfer

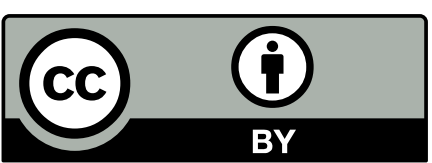

Dieser Text erscheint im Open Access und ist lizenziert unter einer Creative Commons Namensnennung 4.0 International Lizenz.

This is an open access article licensed under a

Creative Commons Attribution 4.0 International License.

veröffentlicht / first published: 01/04/2005

zuletzt geändert / last updated: 15/01/2010 


\title{
Twelve-Tone Theory
}

\author{
Christoph Neidhöfer
}

Im Unterschied zum deutschsprachigen Gebrauch besteht in der amerikanischen (und allgemein angelsächsischen) Musiktheorie keine klare Trennung zwischen den Begriffen Zwölftonmusik ('twelve-tone music`) und serielle Musik ('serial musicı). Während jedes im deutschsprachigen Sinne - zwölftönige Werk als sserials, stwelve-tone` oder sdodecaphonic bezeichnet wird, schließen die Begriffe stwelve-tone sik mit ein, in der das Reihenprinzip auch außerhalb der Tonhöhenstruktur (insbesondere im Bereich des Rhythmus) zur Anwendung kommt. Wo eine klare Unterscheidung zwischen (im deutschsprachigen Sinne) seriellen und zwölftönigen Techniken erforderlich ist, werden erstere in der englischen Terminologie allgemein durch die Begriffe integral serialism` oder stotal serialism p präzisiert. Die englischen Termini sserial und sserialization bezeichnen die Konstruktion durch Reihen (sseries`) allgemein.

Die Beschäftigung der amerikanischen Musiktheorie mit der Dodekaphonik der Zweiten Wiener Schule setzt in den 1930er Jahren ein. In seinem 1936 publizierten Artikel "Schoenberg's Tone-Rows and the Tonal System of the Future« rezipiert Richard S. Hill (1901-1961) die deutsch-, französisch- und englischsprachige Literatur zur atonalen und zwölftönigen Musik, faßt die Zwölftontheorien von Josef Matthias Hauer und Herbert Eimert zusammen und erstellt eine Übersicht über die Verwendung der Zwölftontechnik in Schönbergs bisherigen Werken'. Bei allem Respekt gegenüber Schönbergs Musik bemängelt Hill, daß es oft schwer sei, Schönbergs Reihenstrukturen hörend nachzuvollziehen. Hill argumentiert, daß das Abspinnen einer Zwölftonreihe in etwa dem Spielen einer (diatonischen) Tonleiter entspricht, deren skalenartige Anordnung der Töne nicht deren gegenseitige funktionale Beziehungen abbildet. Deshalb sei Schönbergs Musik so schwer verständlich. Im diatonischen System hält Hill fest, daß beispielsweise die Anordnung der Töne der C-Dur-Skala als C-G-E-F-A-H-D-C die funktionalen Beziehungen darstellt, welche man häufig in tonaler Musik antrifft. Diese Anordnung nennt er einen sfunctional mode tonreihen entwickelt werden könnten. Hill, der selbst nicht Komponist war und deshalb seine Ideen nicht praktisch umsetzen konnte, spekuliert, daß sich im Laufe der Zeit bestimmte Zwölftonreihen als sunctional modes` durch so etwas wie eine historische Selektion herauskristallisieren würden, so daß schließlich einer Hauptreihe eine Anzahl untergeordneter Reihen zur Seite stünden. Sobald sich eine Zwölftonreihe als smodes etabliert hätte, bestünde auch keine Notwendigkeit mehr, daß die Reihe als Ganzes an

1 Op. 23,5 bis op. 35, vgl. Hill 1936.

ZGMTH 2/2-3 (2005) | 207 
der musikalischen Oberfläche erkennbar sein müßte. Hill schlägt außerdem vor, daß solche zwölftönigen ımodes` in der Art des vortonalen Kontrapunkts kompositorisch umgesetzt werden könnten.

Hills Artikel nimmt eine zentrale Stellung in der Entwicklung der amerikanischen Twelve-Tone Theory ein. Ernst Krenek (1900-1991), 1938 emigriert und mit Hills Artikel seit seinem Erscheinen vertraut ${ }^{2}$, entwickelte ausgehend von Hills Ideen seine Technik (und Theorie) der rotierenden Hexachorde, die er erstmals in seinem Chorwerk Lamentatio Jeremiae Prophetae (1941-42) anwendete. Die Töne innerhalb jeder der beiden Hälften (Hexachorde) der Zwölftonreihe werden systematisch rotiert, indem sukzessive der jeweils erste Ton ans Ende versetzt wird. Aus dem ersten Hexachord F-G-A-B-Des-Es entstehen G-A-B-Des-Es-F, A-B-Des-Es-F-G usw., aus dem zweiten Hexachord H-C-D-E-Fis-Gis folgen C-D-E-Fis-Gis-H, D-E-Fis-Gis-H-C usw. Die sechs Rotationen eines jeden Hexachords nennt Krenek »diatonic», weil sie alle dieselben sechs Töne enthalten. In einem zweiten Schritt transponiert er alle sechs Rotationen eines Hexachords derart, daß sie alle mit dem ersten Ton des ursprünglichen Hexachords beginnen. Im erwähnten Falle lauten die transponierten Rotationen des ersten Hexachords F-G-A-B-Des-Es (ursprüngliches Hexachord), F-G-As-Ces-Des-Es, F-Fis-A-H-CisDis usw. Diese nennt er »chromatic«, weil sie zusammen das chromatische Total produzieren. ${ }^{3}$ Dadurch, daß die beiden Hexachorde der Zwölftonreihe individuell rotiert und transponiert werden, verschwindet die Reihe als Einheit von der musikalischen Oberfläche; in Kreneks Terminologie ist die Zwölftonreihe nun nicht mehr »motival« (d. h. in ihrer originalen motivischen Struktur) verwendet, sondern »extra-motival« (d. h. aus dem Hintergrund operativ) eingesetzt.

Die Idee, die Reihe sextra-motival zu verwenden, steht auch im Zentrum der Theorie von George Perle (*1915), der bei Krenek studierte, aber laut eigener Aussage seine Theorie unabhängig von Hills Artikel entwickelte. ${ }^{4}$ Perles Version der stwelve-tone modality - am ausführlichsten in seinem Buch Twelve-Tone Tonality (1977) zusammengefaßt - geht aus von der Überlagerung zweier verschachtelter Zyklen von Quinten. Ein Zyklus absteigender Quinten (im folgenden durch Kleinbuchstaben angedeutet) wird einem Zyklus aufsteigender Quinten (Großbuchstaben) einverleibt: C-c-G-f-D-b-Aes-E-as-H-des-Fis-fis-Cis-h-As-e-Es-a-B-d-F-g-C-c. Das Resultat nennt Perle ein »cyclic set«. Dieses `cyclic set` wird dann mit einer Rotation einer der zwölf Transpositionen seiner Umkehrung Note-gegen-Note kombiniert. Eine solche Rotation einer (transponierten) Umkehrung lautet beispielsweise $\mathrm{E}-\mathrm{C}-\mathrm{A}-\mathrm{g}-\mathrm{D}-\mathrm{d}-\mathrm{G}-\mathrm{a}-\mathrm{C}-\mathrm{e}-\mathrm{F}-\mathrm{h}-\mathrm{B}-\mathrm{fis}-\mathrm{Es}-$ cis-As-as-Cis-es-Fis-b-H-f-E. ${ }^{5}$

Perle faßt jeweils drei benachbarte Töne im scyclic setı und die drei Töne, die an entsprechender Stelle in der zugeordneten Umkehrung auftreten, zusammen. Die einzelnen Töne der resultierenden Sechstongruppe - oft treten dabei auch Tonrepetitionen

2 Krenek 1937, 57-58, 1939, 199, 1943, 82.

3 Krenek 1943, 91, 1962, 74-75.

4 Perle 1941, 275.

5 Diese Tonfolge spiegelt das `cyclic set` um die Achse Cis oder G, mit einer Rotation um vier Positionen nach rechts. 
auf - setzt Perle kompositorisch in freier Reihenfolge ein. Die ursprüngliche motivische Struktur des ‘cyclic set` ist somit in der kompositorischen Realisation nicht mehr direkt erkennbar, sondern nunmehr sextra-motival wirksam.

Die Erforschung der strukturellen Eigenschaften des Zwölftonsystems mit Blick über die rein melodisch-motivische Struktur der Zwölftonreihe hinaus prägt auch die weitere Entwicklung der amerikanischen Twelve-Tone Theory. In seiner 1946 vollendeten (aber erst 1992 von der Princeton University angenommenen) Dissertation mit dem Titel »The Function of Set Structure in the Twelve-Tone System « untersucht Milton Babbitt (*1916) das Zwölftonsystem mit Hilfe der mathematischen sset theory< und sgroup theory`. Jeder Ton einer Zwölftonreihe kann als ein Zahlenpaar in der Form ' $x, y<$ dargestellt werden, in dem $\mathrm{x}$ die Position (von 0 bis 11) innerhalb der Reihe und y die Tonhöhenqualität unabhängig von ihrem spezifischen Register (d. h. eine der spitch classes`C $=0, C$ is $=1, \ldots$ $\mathrm{H}=11$ ) bezeichnet. ${ }^{6}$ Jede Zwölftonoperation (z. B. Umkehrung, Krebs, Krebsumkehrung) kann dann als eine auf die zwölf Zahlenpaare einer Reihe anzuwendende Operation definiert werden. ${ }^{7}$

Babbitt erforscht dabei das Zwölftonsystem insbesondere unter zwei Aspekten. Zum einen erörtert er, unter welchen Umständen ein Teil einer Reihe mit Teilen einer oder mehrerer ihrer Zwölftontransformationen so kombiniert werden kann, daß wiederum das chromatische Total ausgeschöpft ist. Diese Eigenschaft nennt er »combinatoriality«. Zum anderen zeigt Babbitt, wie aus einer Zwölftonreihe andere Reihen abgeleitet werden können, die mit der ursprünglichen Reihe gewisse kombinatorische oder motivische Eigenschaften teilen (»secondary sets« und »derived sets«). Ausgangspunkt für Babbitts Fragestellungen ist sein Studium der Musik der Zweiten Wiener Schule, insbesondere der Dodekaphonik Schönbergs, deren System nach seiner Einschätzung bis in die 1940er Jahre hinein nur rudimentär verstanden wurde und in seinem vollen Potential kaum ausgeschöpft war. Babbitt betrachtet das Zwölftonsystem unabhängig von historischen und stilistischen Fragen, die er für das Studium des Systems selbst für irrelevant hält. ${ }^{8}$

`Combinatoriality ist eine Eigenschaft, in der einer Zwölftonreihe eine ihrer Transformationen (Umkehrung, Krebs, Krebsumkehrung) so zugeordnet wird, daß die Töne der ersten Hälfte der Reihe zusammen mit den Tönen der ersten Hälfte ihrer Transformation das chromatische Total (`aggregate $`$ ) ergeben. ${ }^{9}$ Wichtig ist in diesem Zusammenhang, daß Babbitt die beiden Hälften der Zwölftonreihe - die shexachords` - als sunordered sets` (also unabhängig von der Reihenfolge der Töne innerhalb der Reihenhälften) betrachtet. Babbitt unterscheidet dabei grundsätzlich zwei Arten von ıcombinatorialityı. Diejenigen shexachordsı, die sich entweder mit einer Transposition oder mit einer Umkehrung ihrer selbst zum saggregate` ergänzen lassen, nennt er ısemi-combinatoriak.

6 Babbitt 1946, 8.

7 In der Umkehrung bleiben beispielsweise alle $x$-Werte unverändert, während alle y-Werte durch den Wert z-y ersetzt werden, wobei z eine Konstante ist.

8 Darin liegt der Hauptpunkt seiner Kritik an den Schriften von René Leibowitz zur Zwölftontechnik der Wiener Schule, Babbitt 1950.

9 Aus dieser Eigenschaft folgt, daß dasselbe auch für die zweite Hälfte der Reihe und ihrer Transformation zutrifft. 
Letztere Eigenschaft trifft insbesondere auf viele von Schönbergs Reihenhälften zu. Diejenigen shexachords`, die sich auf ihr >complementı (d.h. die sechs Töne, die das jeweilige shexachord zum saggregate ergänzen) gleichzeitig durch Transposition und (transponierte) Umkehrung abbilden lassen, nennt er sall-combinatoriak. Diese Eigenschaft ist in nur sechs Fällen erfüllt. Die entsprechenden shexachords`, auch ısource sets، genannt, lauten (so transponiert, daß sie alle mit $C$ beginnen): (1) $C-C i s-D-D i s-E-F$, das chromatische shexachord; (2) C-D-Dis-E-F-G; (3) C-D-E-F-G-A, das diatonische shexachord;; (4) C-Cis-D-Fis-G-Gis; (5) C-Cis-E-F-Gis-A, mit Bezug auf die Reihe aus Schönbergs op. 41 später in der amerikanischen Musiktheorie oft auch ১Ode to Napoleon hexachord genannt; (6) C-D-E-Fis-Gis-Ais, die Ganztongruppe.

Das Prinzip der >combinatoriality، läßt sich auch auf die Kombination von kürzeren Segmenten einer Reihe anwenden. Beispielsweise ist es möglich, drei der 48 Formen einer (entsprechend ausgewählten) Reihe so zu überlagern, daß die ersten Viertongruppen aus jeder der drei Reihenformen sich zum chromatischen Total ergänzen, während dasselbe Prinzip auch auf die jeweils zweiten und dritten Viertongruppen zutrifft. Eine solche Anordnung mehrerer Reihen - es können auch verschiedene Reihen sein - nennt Babbitt »array«. Die Unterteilung der Reihe in kleinere Segmente nennt er »partitioning«. Die im sarray überlagerten Reihen können auch unregelmäßig segmentiert sein (z. B. als $1+5+3+3$ Töne).

Die Idee, aus einer Zwölftonreihe andere Reihen abzuleiten, hängt - abgesehen vom Studium der Bergschen Reihenableitungen - eng mit der Rezeption der Theorien von Hill und Perle zusammen, die die Reihe als ein aus dem Hintergrund wirkendes, nicht unbedingt wörtlich an der musikalischen Oberfläche erscheinendes Element begreifen. Babbitt unterscheidet zwei Arten der Ableitung: (1) Aus einer Zwölftonreihe (`row sset`) läßt sich ein sogenanntes ssecondary setı herstellen, indem man den Tonvorrat innerhalb der beiden Reihenhälften (oder in jedem Drittel, den stetrachords`, jedem Viertel, den strichords`, oder jedem Sechstel, den `dyads`) neu ordnet. Dadurch entsteht eine neue melodisch-motivische Struktur, aber die kombinatorischen Eigenschaften der ursprünglichen und abgeleiteten Reihe bleiben dieselben (da `combinatoriality sich auf ungeordnete Gruppen bezieht). (2) Aus einer Zwölftonreihe läßt sich ein sogenanntes `derived set ableiten, indem aus der ursprünglichen Reihe ein Segment von zwei bis sechs Tönen isoliert wird, welches dann mit Transpositionen und / oder Umkehrungen seiner selbst zu einer neuen Zwölftonreihe ergänzt wird.

Babbitt wendet seine Twelve-Tone Theory im weiteren im Bereich des Rhythmus an. Ausgehend von der Tatsache, daß das chromatische System modular ist - die zwölf Tonhöhenqualitäten (`pitch classes`) wiederholen sich in jeder Oktave -, übersetzt Babbitt die numerische Darstellung einer Tonhöhenstruktur in sogenannte stime pointsı: In einer metrischen Struktur von zwölf stime points` (z. B. den zwölf Sechzehnteln in einem 3/4-Takt) werden die zwölf Positionen von 0 bis 11 durchnumeriert. Jeder Tonhöhe kann dann die ihrer spitch-classı-Nummer entsprechende Position zugeordnet werden. Die Art der numerischen Folge ist dabei von zentraler Bedeutung. Folgt nämlich auf eine Zahl eine kleinere Zahl, wird die kleinere Zahl in den nächsten Takt verlegt (da man ja sonst plötzlich rückwärts hören müßte); Babbitts System beinhaltet also nicht eine 
Übersetzung der Frequenzverhältnisse im Tonhöhenbereich in rhythmische Dauernproportionen. Babbitts ıtime-pointı-System ermöglicht es im weiteren, die traditionellen Zwölftonoperationen (Umkehrung, Krebs, Krebsumkehrung) im rhythmischen Bereich anzuwenden.

Babbitt kommt auch das Verdienst zu, als einer der ersten die Reihentechnik von Igor Strawinsky theoretisch untersucht zu haben, insbesondere die Technik der rotierenden Hexachorde, welche Strawinsky von Krenek übernommen hatte und auch auf rotierende Tetrachorde übertrug. ${ }^{10}$ Babbitts Zwölftontheorien wurden von einer Anzahl jüngerer Theoretiker und Komponisten aufgegriffen und erweitert. In seinem Artikel von 1961, »The Source Set and Its Aggregate Formations", zeigt Donald Martino (*1931), unter welchen Umständen aus bestimmten Sechstongruppen, Viertongruppen und Dreitongruppen (den ısource sets`) das chromatische Total generiert werden kann. Die verschiedenen Konstellationen werden als ımosaics` bezeichnet. Brian Alegant, Andrew Mead, Robert Morris und Richard Kurth widmeten, unter anderen, den ımosaics` und spartitions`später weitergehende Studien. ${ }^{11}$ Babbitts Untersuchungen zu Strawinskys Technik der rotierenden Hexachorde und weitere Studien zu diesem Thema, insbesondere von Claudio Spies und John Rogers, führten schließlich zu Robert Morris' eingehender Studie über allgemeine Systeme rotierender sarrays.${ }^{12}$ Der Artikel »A Theory of Segmental Association in Twelve-Tone Music« von David Lewin (1933-2003) und die Dissertation des schwedisch-kanadischen Theoretikers Bo Alphonce (1931-2000) schließen sich an Babbitts theoretische Untersuchungen über sinvariants` an. ${ }^{13}$ Ausgehend von Babbitts Theorie der shexachords` zeigt Lewin in »A Study of Hexachord Levels in Schoenberg's Violin Fantasy« (1967), wie in Schönbergs Werk die verwendeten Formen der Reihe sich in sogenannte sareas` einteilen lassen. Formen der Reihe, die aus denselben zwei komplementären shexachords` (unabhängig von ihren internen Ordnungen) bestehen, bilden eine sarea`. Wie Lewins Analyse aufzeigt, kommt der Folge der verschiedenen sareas eine formbildende Funktion im Werk zu. Robert Morris greift Babbitts rhythmische Theorien auf und zeigt, wie die traditionellen Zwölftonoperationen auch auf jede beliebige, nicht unbedingt zwölftönige Reihe von stime points` angewendet werden können. ${ }^{14}$

Aus der Fülle weiterer Ansätze in der amerikanischen Twelve-Tone Theory seien hier stellvertretend noch folgende herausgegriffen: In ihrem Artikel »The Structure of AllIntervall Series« (1974) haben Robert Morris und Daniel Starr die Strukturen der 3856 (transpositorisch normalisierten) Allintervallreihen auf 267 »source all-interval series« zurückgeführt. In »A Theory of Twelve-Tone Meter« (1984) untersucht Martha Hyde die Beziehungen zwischen der Reihentechnik und der metrischen Struktur in Schönbergs Musik. In seinen späteren Arbeiten - vor allem im Rahmen der von ihm begründeten stransformational theory« - lenkt sich David Lewins Interesse vermehrt auf Strukturen

10 Vgl. Babbitt 1964, 1986, 1987 b.

11 Mead 1988, Morris und Alegant 1988, Kurth 1992, 1993, 1999, Alegant 2001.

12 Spies 1965a, 1965b, 1967, Rogers 1968, Morris 1988.

13 Geordnete oder ungeordnete Tongruppen, die unter bestimmten Transformationen unverändert bleiben, Babbitt 1960, Lewin 1962, Alphonce 1974.

14 Morris 1987, 299-312. 
außerhalb der eigentlichen Zwölftonfaktur. So untersucht er beispielsweise in seiner Analyse des "Simbolo« aus Luigi Dallapiccolas Quaderno musicale di Annalibera (1952) das Zusammenwirken dreier durchweg prominent hörbarer Motive, die aber als solche nicht direkt in der Zwölftonreihe des Werkes auftreten (Lewin 1993). Verschiedene Theoretiker haben Zwölftonstrukturen auch mit Hilfe sogenannter `Klumpenhouwer-networks ( KK-nets ) analysiert. ${ }^{15}$,K-nets vergleichen die Intervallstrukturen von verschiedenen Tonhöhengruppen (ısets`), indem sie ein Netzwerk von Beziehungen definieren, welche auf jedes der verschiedenen ssetsı zutreffen. Dadurch kann beispielsweise gezeigt werden, wie verschiedene Komponenten (unterschiedliche ssetsı) innerhalb einer Zwölftonstruktur durch gemeinsame Eigenschaften aufeinander bezogen sind. Gelegentlich ist das Zwölftonsystem auch in Verbindung mit anderen Tonsystemen untersucht worden. Brian Alegant und John Levey haben beispielsweise kürzlich das Zusammenwirken von Oktatonik und Zwölftontechnik anhand von Dallapiccolas Musik systematisch erforscht (Alegant und Levey, in Vorbereitung).

Eine umfassende Darstellung der amerikanischen Twelve-Tone Theory findet sich in Robert Morris' Buch Composition with Pitch Classes (1987), das sich auch mit der atonalen Theorie befaßt (siehe Summary zur 'Set Theoryı). John Rahns Basic Atonal Theory (1980) ist ein weiterer sKlassiker der amerikanischen Twelve-Tone Theory (der sich wiederum auch mit der atonalen Theorie beschäftigt). Wenn es die Darstellung äußerlich auch nicht vermuten läßt, ist Morris' Buch in erster Linie für Komponierende geschrieben. Ebenfalls für Komponierende verfaßt, aber wiederum auch für Theoretiker interessant ist Charles Wuorinens Simple Composition (1979), eine Anleitung zur Zwölftonkomposition, die von Babbitts Theorien ausgeht. Joseph N. Straus' Introduction to Post-Tonal Theory (2005) vermittelt eine klare Darstellung der Twelve-Tone Theory (und sset theory`) für den Unterricht. Anders als im deutschsprachigen Raum wurde die Zwölftontechnik in den Vereinigten Staaten und Kanada - vor allem im akademischen Umfeld der Universitäten - bis in die 1990er Jahre von Komponisten und Komponistinnen noch sehr häufig verwendet. Wie John Covach in seinem Artikel über die Twelve-Tone Theory in der Cambridge History of Western Music Theory festhält, haben sich Komponierende (wohl als Reaktion gegen die Moderne) allerdings von der Twelve-Tone Theory seit den 1990er Jahren zusehends abgewendet. ${ }^{16}$ Das Wirkungsfeld der Twelve-Tone Theory liegt deshalb heute vor allem im Bereich der Analyse älteren Repertoires.

15 Z.B. Klumpenhouwer 1991, 1998, Straus 1999.

16 Covach 2002, 625. 


\section{Literatur}

Alegant, Brian (1996), „Unveiling Schoenberg's Op. 33b«, Music Theory Spectrum 18, 143-66.

(2001), „Cross-Partitions as Harmony and Voice Leading in Twelve-Tone Music», Music Theory Spectrum 23, 1-40.

und Robert Morris (1988), »The Even Partitions in Twelve-Tone Music», Music Theory Spectrum 10, 74-101.

- und John Levey, "Six of One and Half a Dozen of the Other: Octatonicism in Dallapiccola's Twelve-Tone Music«, Music Analysis, in Vorbereitung.

Alphonce, Bo (1974), »The Invariance Matrix«, Ph.D.-Dissertation, Yale University.

Babbitt, Milton (1946), »The Function of Set Structure in the Twelve-Tone System«, Ph.D.-

Dissertation, Princeton University, 1992 angenommen.

— (1950), „Review of Schoenberg et son école and Qu'est-ce que la musique de douze sons? by René Leibowitz«, Journal of the American Musicological Society 3, 57-60, und Babbitt 2003, 10-15.

— (1955), "Some Aspects of Twelve-Tone Composition«, The Score and I.M.A. Magazine 12, 53-61, und Babbitt 2003, 38-47.

— (1960), »Twelve-Tone Invariants as Compositional Determinants«, Musical Quarterly 46, 246-59, auch abgedruckt in: Problems of Modern Music, hg. von Paul Henry Lang, New York: W. W. Norton, 1962, 72-94, und Babbitt 2003, 55-69.

_ (1961), „Set Structure as a Compositional Determinant«, Journal of Music Theory 5, 72-94, Boretz und Cone 1972, 129-47, und Babbitt 2003, 86-108.

— (1962), »Twelve-Tone Rhythmic Structure and the Electronic Medium«, Perspectives of New Music 1, 49-79, Boretz und Cone 1972, 148-79, und Babbitt 2003, 109-140.

- (1964), »Remarks on the Recent Stravinsky«, Perspectives of New Music 2, 35-55, Boretz und Cone 1972, 165-85, und Babbitt 2003, 147-71.

- (1968), »Three Essays on Schoenberg: Concerto for Violin and Orchestra, Das Buch der hängenden Gärten, and Moses and Aaron", in Boretz und Cone 1972, 47-60, und Babbitt 2003, 222-36.

- (1974), "Since Schoenberg", Perspectives of New Music 12, 3-28, und Babbitt 2003, 310-34.

_ (1986), „Order, Symmetry, and Centricity in Late Stravinsky«, in: Confronting Stravinsky, hg. von Jann Pasler, Berkeley: University of California Press, 247-61.

_ (1987a), Words About Music, hg. von Stephen Dembski und Joseph N. Straus, Madison: University of Wisconsin Press.

— (1987b), "Stravinsky's Verticals and Schoenberg's Diagonals: A Twist of Fate», in: Stravinsky Retrospectives, hg. von Ethan Haimo und Paul Johnson, Lincoln: University of Nebraska Press, auch in Babbitt 2003, 404-27.

— (2003), The Collected Essays of Milton Babbitt, hg. von Stephen Peles, Stephen 
Dembski, Andrew Mead und Joseph N. Straus, Princeton: Princeton University Press. Batstone, Philip N. (1972), »Multiple Order Functions in Twelve-Tone Music«, Perspectives of New Music 10, 60-71, 11, 92-111.

Boretz, Benjamin und Edward T. Cone (Hg.) (1968), Perspectives on Schoenberg and Stravinsky, Princeton: Princeton University Press.

- (1972), Perspectives on Contemporary Music Theory, New York: W. W. Norton.

Covach, John (2002), „Twelve-Tone Theory«, in: The Cambridge History of Western Music Theory, hg. von Thomas Christensen, Cambridge: Cambridge University Press, 603-27.

Dubiel, Joseph (1990-92), »Three Essays on Milton Babbitt«, Perspectives of New Music 28, 216-61, 29, 90-123, 30, 82-131.

Eimert, Herbert (1924), Atonale Musiklehre, Leipzig: Breitkopf und Härtel.

Forte, Allen (1973), The Structure of Atonal Music, New Haven: Yale University Press.

Haimo, Ethan und Paul Johnson (1984), »Isomorphic Partitioning and Schoenberg's Fourth String Quartet«, Journal of Music Theory 28, 47-72.

Hauer, Josef M. (1923), Lehrbuch der Zwölftontechnik. Vom Wesen des Musikalischen, Berlin-Lichterfelde: Schlesinger.

— (1925), Vom Melos zur Pauke. Eine Einführung in die Zwölftonmusik, Wien: Universal Edition.

— (1926), Zwölftontechnik. Die Lehre von den Tropen, Wien: Universal Edition.

Hill, Richard S. (1936), "Schoenberg's Tone-Rows and the Tonal System of the Future», Musical Quarterly 22, 14-37.

Hush, David (1982-83/1983-84), »Asynordinate Twelve-Tone Structures: Milton Babbitt's Composition for Twelve Instruments", Perspectives of New Music 21, 152-208, 22, 103-16.

Hyde, Martha M. (1984), „A Theory of Twelve-Tone Meter«, Music Theory Spectrum 6, 14-51.

Kassler, Michael (1967), »Toward a Theory That Is the Twelve-Note-Class System«, Perspectives of New Music 5, 1-80.

Klumpenhouwer, Henry (1991), »A Generalized Model of Voice-Leading for Atonal Music«, Ph.D.-Dissertation, Harvard University.

— (1998), „The Inner and Outer Automorphisms of Pitch-Class Inversion and Transposition. Some Implications for Analysis with Klumpenhouwer Networks", Intégral 12, 81-93.

Kowalski, David (1987), »The Construction and Use of Self-Deriving Arrays«, Perspectives of New Music 25, 286-361.

Krenek, Ernst (1937), Über neue Musik: sechs Vorlesungen zur Einführung in die theoretischen Grundlagen, Wien: Ringbuchhandlung.

_ (1939), Music Here and Now, New York: W. W. Norton.

— (1940), Studies in Counterpoint, New York: G. Schirmer. 
_ (1943), »New Developments of the Twelve-Tone Technique«, Music Review 4, 81-97.

_ (1962), „Extents and Limits of Serial Techniques«, in: Problems of Modern Music, hg. von Paul Henry Lang, New York: W. W. Norton, 72-94.

Kurth, Richard (1992), »Mosaic Polyphony. Formal Balance, Imbalance, and Phrase Formation in the Prelude of Schoenberg's Suite, op. 25«, Music Theory Spectrum 14, 188-208.

- (1993), „Mosaic Isomorphism and Mosaic Polyphony. Balance and Imbalance in Schoenberg's Twelve-Tone Rhetoric«, Ph.D.-Dissertation, Harvard University.

- (1999), »Partition Lattices in Twelve-Tone Music. An Introduction«, Journal of Music Theory 43, 21-82.

Lewin, David (1962), »A Theory of Segmental Association in Twelve-Tone Music«, Perspectives of New Music 1, 89-116, und Boretz und Cone 1972, 180-207.

_ (1966), „On Certain Techniques of Reordering in Serial Music», Journal of Music Theory 10, 276-87.

- (1967), „A Study of Hexachord Levels in Schoenberg's Violin Fantasy«, Perspectives of New Music 6, 18-32, auch abgedruckt in: Perspectives on Schoenberg and Stravinsky, hg. von Benjamin Boretz und Edward T. Cone, New York: W. W. Norton, 1972, 78-92.

_ (1987), Generalized Musical Intervals and Transformations, New Haven: Yale University Press.

- (1993), Musical Form and Transformation, New Haven: Yale University Press.

London, Justin (2002), „Rhythm in Twentieth-Century Theory«, in: The Cambridge History of Western Music Theory, hg. von Thomas Christensen, Cambridge: Cambridge University Press, 695-725.

Martino, Donald (1961), »The Source Set and Its Aggregate Formations «, Journal of Music Theory 5, 224-71.

Mead, Andrew (1983), „Detail and the Array in Milton Babbitt's My Complements to Roger", Music Theory Spectrum 5, 89-109.

- (1988), „Some Implications of the Pitch Class / Order Number Isomorphism Inherent in the Twelve-Tone System, Part One«, Perspectives of New Music 26, 96-163.

— (1989), »Twelve-Tone Organizational Strategies. An Analytical Sampler«, Intégral 3, 93-170.

(1994), An Introduction to the Music of Milton Babbitt, Princeton: Princeton University Press.

Morris, Robert (1977), „On the Generation of Multiple Order Function Twelve-Tone Rows", Journal of Music Theory 21, 238-63.

- (1983-84), „Set-Type Saturation among Twelve-Tone Rows«, Perspectives of New Music 22, 187-217.

- (1987), Composition with Pitch-Classes. A Theory of Compositional Design, New Haven: Yale University Press. 
_ (1988), »Generalizing Rotational Arrays«, Journal of Music Theory 32, 75-132.

(2003), »Pitch-Class Duplication in Serial Music. Partitions of the Double Aggregate", Perspectives of New Music 41, 96-121.

Morris, Robert und Daniel Starr (1974), »The Structure of All-Interval-Series«, Journal of Music Theory 18, 364-89.

(1977-78), »A General Theory of Combinatoriality and the Aggregate«, Perspectives of New Music 16, 3-35, 50-84.

Nolan, Catherine (2002), »Music Theory and Mathematics", in: The Cambridge History of Western Music Theory, hg. von Thomas Christensen, Cambridge: Cambridge University Press, 272-304.

Peles, Stephen (1998), „Serialism and Complexity«, in: The Cambridge History of American Music, hg. von David Nicholls, Cambridge: Cambridge University Press, 496-516.

Perle, George (1941), »Evolution of the Tone-Row. The Twelve-Tone Modal System«, Music Review 2, 273-87.

— (1954), »The Possible Chords in Twelve-Tone Music», Score 9, 54-58.

- (1962), Serial Composition and Atonality. An Introduction to the Music of Schoenberg, Berg and Webern, Berkeley: University of California Press.

- (1977), Twelve-Tone Tonality, Berkeley: University of California Press.

— (1990), The Listening Composer, Berkeley: University of California Press.

Rahn, John (1980), Basic Atonal Theory, New York: G. Schirmer.

Rogers, John (1967), „Towards a System of Rotational Arrays", Proceedings of the American Society of University Composers 2, 61-84.

_ (1968), „Some Properties of Non-Duplicating Rotational Arrays«, Perspectives of New Music 7, 80-102.

Rothgeb, John (1967), „Some Ordering Relationships in the Twelve-Tone System«, Journal of Music Theory 11, 176-97.

Rothstein, William (1980), »Linear Structure in the Twelve-Tone System. An Analysis of Donald Martino's Pianississimo«, Journal of Music Theory 24, 129-65.

Schoenberg, Arnold (1936), „Schoenberg's Tone-Rows«, übers. von Leo Black, in: Style and Idea. Selected Writings of Arnold Schoenberg, hg. von Leonard Stein, Berkeley und Los Angeles: University of California Press, 1984, 213-14.

- (1941), „Composition with Twelve Tones (1)«, übers. von Leo Black, in: Style and Idea. Selected Writings of Arnold Schoenberg, hg. von Leonard Stein, Berkeley und Los Angeles: University of California Press, 1984, 214-44.

— (ca. 1948), „Composition with Twelve Tones (2)«, übers. von Leo Black, in: Style and Idea. Selected Writings of Arnold Schoenberg, hg. von Leonard Stein, Berkeley und Los Angeles: University of California Press, 1984, 245-49.

Schubert, Peter (1994), «A Multidetermined Moment in Milton Babbitt's Three Cultivated Choruses «", Theory and Practice 19, 57-82.

Spies, Claudio (1965a), »Some Notes on Stravinsky's Abraham and Isaac«, Perspectives 
of New Music 3, 104-26, und Boretz und Cone 1968, 186-209.

(1965b), „Some Notes on Stravinsky's Variations», Perspectives of New Music 4, 62-74, und Boretz und Cone 1968, 210-22.

_ (1967), »Some Notes on Stravinsky's Requiem Settings", Perspectives of New Music 5, 98-123, und Boretz und Cone 1968, 223-50.

— (1974), »Vortrag / 12 TK / Princeton«, Perspectives of New Music 13, 58-136.

Stanfield, Michael (1984), „Some Exchange Operations in Twelve-Tone Theory. Part One«, Perspectives of New Music 23, 258-77.

Stewart, John L. (1991), Ernst Krenek. The Man and his Music, Berkeley: University of California Press.

Straus, Joseph N. (1999), „Stravinsky's `Construction of Twelve Verticals». An Aspect of Harmony in the Serial Music", Music Theory Spectrum 21, 43-73.

— (32005), Introduction to Post-Tonal Theory, Englewood Cliffs, New Jersey: Prentice Hall.

Westergaard, Peter (1966), »Toward a Twelve-Tone Polyphony«, Perspectives of New Music 4, 90-112, und Boretz und Cone 1972, 238-60.

Winham, Godfrey (1970), „Composition with Arrays«, Perspectives of New Music 9, 43-67, und Boretz und Cone 1972, 261-85.

Wuorinen, Charles (1979), Simple Composition, New York: C. F. Peters.

Yasser, Joseph (1932), A Theory of Evolving Tonality, New York: American Library of Musicology, Nachdruck: New York: Da Capo Press, 1975. 